\title{
AN IRIS MECHANISM DRIVEN TEMPERATURE CONTROL OF SOLAR THERMAL REACTORS
}

\author{
L. Van den Langenbergh, C. Ophoff, N. Ozalp* \\ KU Leuven, Mechanical Engineering Department, Engineering Technology Cluster, 2860 Belgium
}

\begin{abstract}
In spite of their attraction for clean production of fuels and commodities; solar thermal reactors are challenged by the transient nature of solar energy. Control of reactor temperature during transient periods is the key factor to maintain solar reactor performance. Currently, there are few techniques that are being used to accommodate the fluctuations of incoming solar radiation. One of the commonly practiced methods is to adjust the mass flow rate of the feedstock which is very simple to implement. Another method is focusing and defocusing of the heliostats which requires careful control of the heliostat field. Although these techniques are very convenient and widely being practiced successfully, there are several drawbacks associated with each of them. For example, although the temperature inside a solar reactor can be easily controlled by varying the mass flow rate of the feedstock, it disturbs the flow dynamics inside reactor. This is a major problem for cases where the flow pattern must be maintained constant. Therefore, an alternative temperature control inside the reactor should be developed. This paper presents a promising approach where an iris mechanism is driven by a closed loop control system to adjust the area where solar energy enters the reactor.
\end{abstract}

Keywords: Solar radiation, Control system, Iris, Aperture, Solar reactor

\section{INTRODUCTION}

A solar reactor is a vessel housing endothermic chemical reactions driven by concentrated solar energy. Solar reactor is made of several major parts as shown in Figure 1. Concentrated solar energy enters reactor's cavity trough an aperture made of quartz. Feedstock enters through three inlet ports to create enhanced mixing via vortex flow formation. Quartz window is cooled by water to avoid excess heating.

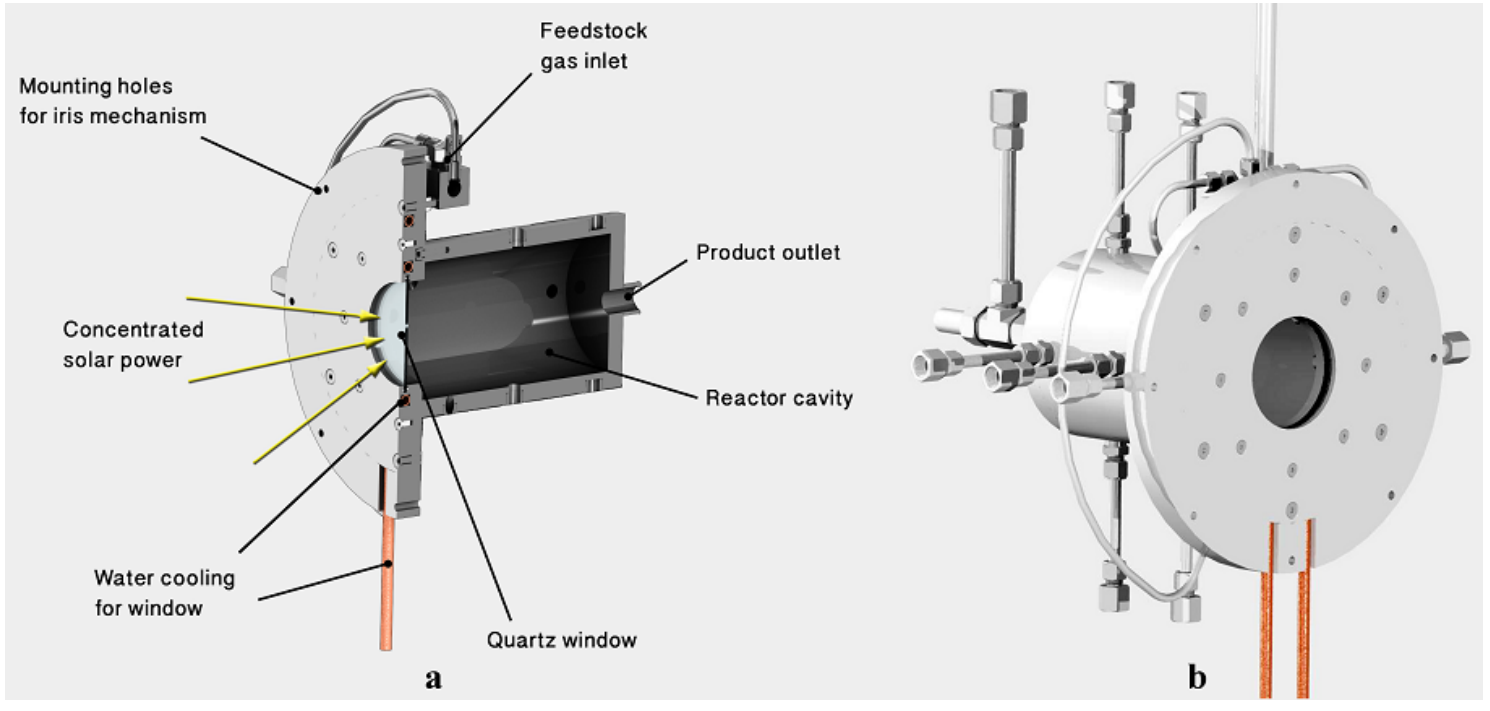

Fig. 1: (a) Cross sectional view of the solar reactor, (b) Solar reactor assembled

\footnotetext{
*Corresponding author: nesrin.ozalp@kuleuven.be
} 


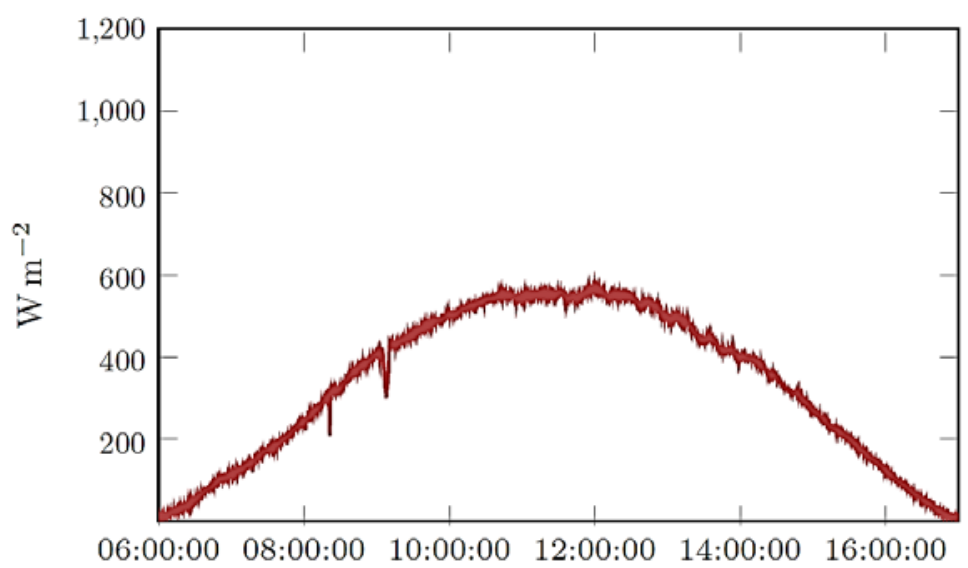

Fig. 2: Gaussian distribution of Global Horizontal Irradiance measured in March 8, 2014 [1]

It is possible to compensate fluctuating nature of solar energy by implementing several control techniques. One of the most applicable techniques is the mass flow adjustment of the feedstock [2-5]. Chemical reactions with higher feedstock flow rate consume more energy reducing the thermal energy stored in the reactor. Therefore, adjustment of flow rates according to the incoming solar radiation is a simple method to maintain semi-constant temperature. However, such control measure may lower the production rate and disturb the flow pattern inside the reactor which would be an issue if specific flow dynamics needs to be maintained [6].
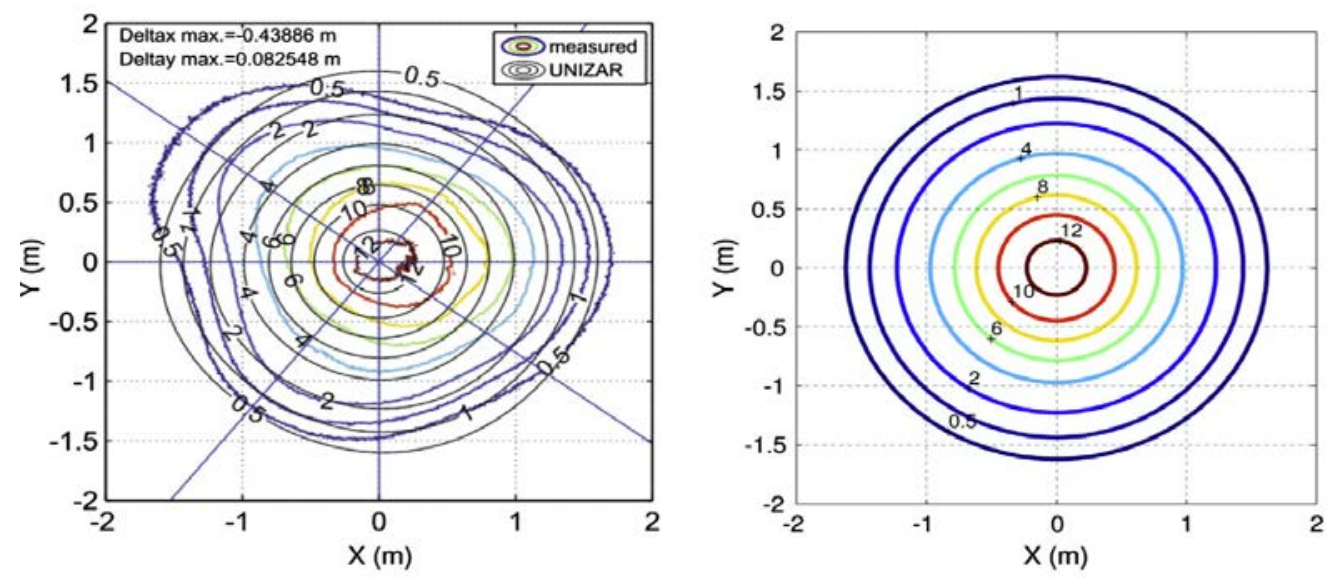

Fig. 3: Flux density according to radius position [7]

Another successful technique to accommodate transient nature of the incoming solar radiation is focus/defocus of the heliostats. Heliostats are mirror-like devices used to reflect sunlight towards solar receiver. Solar reactor temperature can be controlled by decrease or increase in flux density captured by the aperture of the receiver according to the flux distribution given in Figure 3. However, this method has the risk of power loss due to partial use of heliostats. For example, Sack et al. (2012) use this method to run twostep water splitting cycle which requires two different temperatures [8]. It is seen that nearly $60 \%$ power is lost after 12:00 pm for every alternate cycle of the two-step water splitting cycle due to deliberate underutilization of available heliostats. This does not represent an ideal concentrated solar power process plant from a cost perspective because installed heliostats form $50-70 \%$ of the depreciable capital. Therefore, from a cost perspective it is imperative to make full use of the installed capacity of heliostats, irrespective of fluctuation in solar radiation.

This paper presents an iris mechanism solar reactor to accommodate fluctuations in solar energy via a closed loop control system. This technique does not influence the inner flow dynamics of the solar and makes full use of the heliostat field. Following sections describe design of the proposed iris mechanism and the development of a closed loop control system. 


\section{APERTURE CONCEPT}

Previous studies have shown that implementation of fixed aperture reduces the full use of available solar radiation [9-10]. Alternatively, a variable and controllable aperture can be used to adopt fluctuations of incoming solar energy. However, there are set of design requirements to create an iris mechanism. For example, the aperture must approximate a circular shape. Also, it should have a diameter that can compile with the heat transfer requirements to maintain semi-constant temperatures according to the flux levels. A closed loop steering mechanism can ensure correct timing for the aperture’s opening and closure.

Iris mechanisms have been applied in many fields as seen in Table 1. Photography was the pioneering field implemented iris design. Other fields of application came out afterwards and made advancements in iris designs and control. For example, Ref. [11] shows an apparatus used in cameras which controls a variable aperture diaphragm by incorporating a drive motor.

Table 1: Overview and comparison of iris mechanisms used in different fields

\begin{tabular}{|c|c|c|c|}
\hline $\begin{array}{c}\text { Field of } \\
\text { application }\end{array}$ & Photography & Flow Dynamics & Infra-Red Imaging \\
\hline Media Flow & Light & $\begin{array}{l}\text { Fuel mixture } \\
\text { (Air and fluid) }\end{array}$ & IR light \\
\hline $\begin{array}{c}\text { Control } \\
\text { Mechanism }\end{array}$ & $\begin{array}{l}\text { Driving motor and pin-slot } \\
\text { mechanism }\end{array}$ & $\begin{array}{l}\text { Gears, chains } \\
\text { or belts }\end{array}$ & Pin-slot mechanism \\
\hline $\begin{array}{l}\text { Aperture } \\
\text { Structure }\end{array}$ & Several blades & Several blades & $\begin{array}{c}\text { L-and C-Shaped } \\
\text { Blades }\end{array}$ \\
\hline $\begin{array}{l}\text { Component } \\
\text { Motion }\end{array}$ & Rotational & Rotational & $\begin{array}{l}\text { Rotational and } \\
\text { translation }\end{array}$ \\
\hline $\begin{array}{l}\text { Component } \\
\text { Material }\end{array}$ & $N / A$ & $N / A$ & $\begin{array}{l}\text { Stainless steel blades, } \\
\text { titanium actuator }\end{array}$ \\
\hline $\begin{array}{l}\text { Cross-Sectional } \\
\text { Exposure }\end{array}$ & Circular & Circular & Rectangular/Octagonal \\
\hline \multicolumn{4}{|l|}{$\begin{array}{c}\text { Schematic of } \\
\text { Design Concept }\end{array}$} \\
\hline Reference & Yamada, T., et al., 2013 & Cheung, N., 2012 & Gat, N., et al., 2013 \\
\hline
\end{tabular}

Another example iris mechanism seen in Table 1 is being used in flow dynamics field to control supply and exhaust of gases in a conventional combustion engine [12]. Instead of using traditional poppet valves, the inand outlets of the combustion chamber is controlled independently with this mechanism. This leads to better combustion efficiency and, consequently, reduced pollution. Comparable to the field of photography, infrared imaging also uses variable aperture mechanism [13]. This specific application is to avoid unwanted infrared radiation when using thermal IR camera. These applications $[11,13]$ are in fact identical to iris mechanism used in solar reactor concept. However, scalability of these mechanisms is often limited by their components. Therefore small-scale applications of iris mechanisms are created using electro-fluids. For example, such variable aperture concepts have been applied in medicine and biochemistry [14, 15]. 


\section{DESIGN OF AN IRIS MECHANISM}

The main parts of an iris mechanism are moving blades. The opening surface depends on the position and movement of these components. Various aspects should be taken into consideration before designing such mechanisms. Figures $4 \mathrm{a}-4 \mathrm{~d}$ demonstrate that increasing the number of blades results in more circular aperture shape. The less circular the shape, the bigger the drawback in optics and heat transfer [9]. These drawbacks are result of diffraction and a decreased number of wavelengths passing through the aperture.
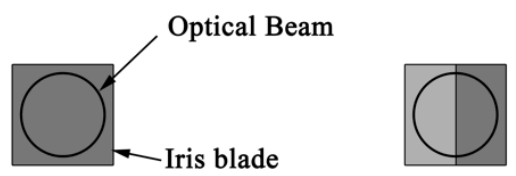

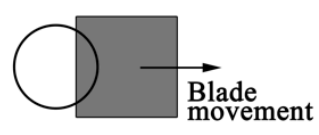

(a)

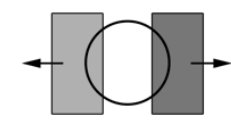

(b)

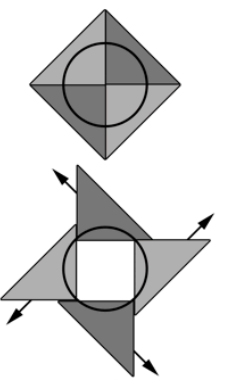

(c)
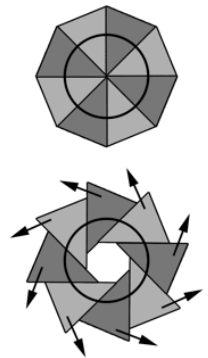

(d)
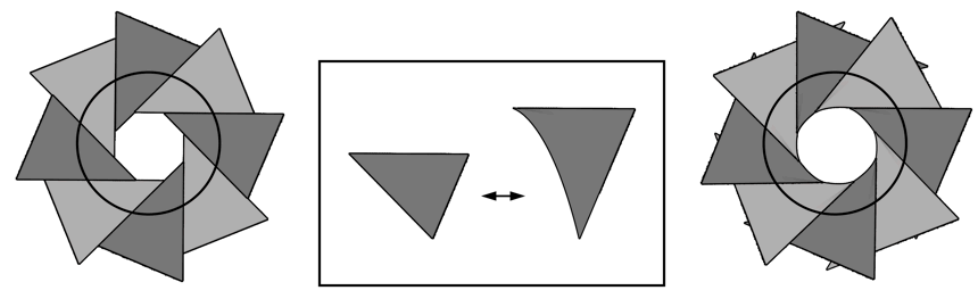

(e)

Fig. 4: (a-d) One, two, four and eight blades iris mechanism, (e) Non-curved versus curved blade. [Based on Ref. 16, Figure 1, p. 1701]

Another important design aspect is the shape of the blade. By using curved edge blades on the inner side of the opening area, the aperture can become more circular. This is shown in Figure 4e where the octagon becomes a circle. Consequently, the figure shows that an iris mechanism featuring an infinite number of curved blades would be the most optimum design. This in turn implies that the blades need to be infinitely thin or at least as thin as possible. But this requirement is limited by the interference of individual blades.

Design of an iris mechanism takes several steps. One of the first steps is the understanding of previous designs to avoid duplicate. Another important point is to ensure manufacturability and practicality. In present work, the design process started with the creation of the blades. Shape of the blades is one of the key parameters determining the aperture opening and subsequently the circularity of the area. Figure 5 shows the blade design of this present work. The blade has two inner edges instead of one and is therefore called a double-sided blade. This feature makes this design different from previous iris designs. Assembled design has eight blades to approximate circular area. It was observed that more than eight blades increased the thickness of the assembly and had the risk of encountering interference issues like experienced with other blades developed by our research group. 


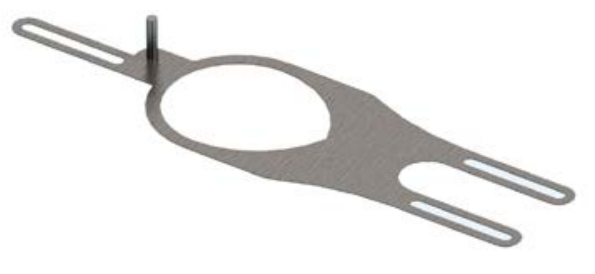

a

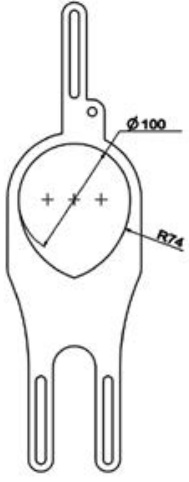

b

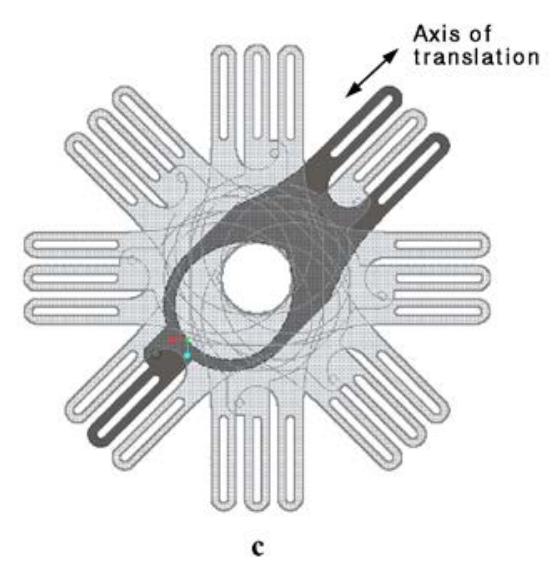

c

Fig. 5: (a) Single blade design, (b) Dimensions, (c) Assembly

Once the blade shape was finalized, the motion mechanism was designed. The motion mechanism was supposed to move the blades to generate a variable opening area. The design was made in such a way that the blades slide towards the center point of the aperture as seen in Figure 5c, e.g. does not require rotation like most of the iris mechanisms. A pin-slot mechanism was applied for motion. This was achieved by using a plate with slots which hereinafter referred to as the actuator or middle plate. Each blade was pin welded perpendicularly with $8 \mathrm{~mm}$ diameter that slides into the slots. When the actuator rotates, these pins experience frictional force with resultant force that forces them to move in the slot. This way, the blades move along their axis of translational movement and open/close the iris mechanism.

Next step was to link the stepper motor to the actuator. Initial idea was to use gears to transfer the rotational motion from the motor to the actuator. However, it was changed to a chain transmission to avoid overheating of the stepper motor because of being exposed to high flux coming from the solar simulator. Use of chain rather than direct gear transmission allows to place the motor over a distance far enough from the heat source. The driving sprocket was welded on a shaft and coupled to the stepper motor using a flexible coupling as shown in Figure 6a.

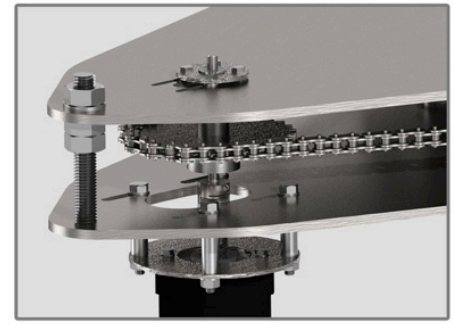

a

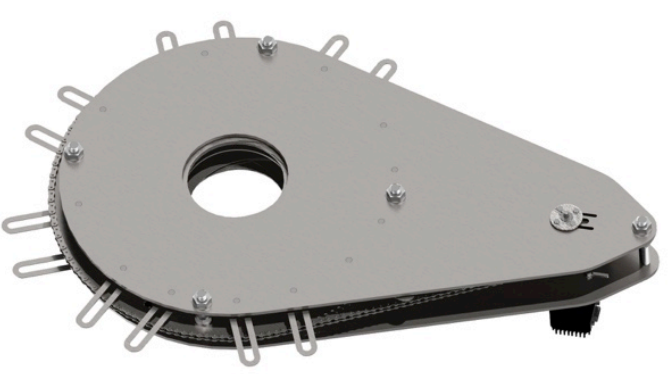

b

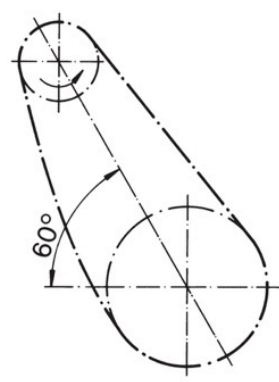

c

Fig. 6: (a) Driving sprocket, (b) Finished CAD model, (c) Drive configuration

Geometric constraints in the design created an issue where the blades' guiding slots sticking out of the assembly as shown in Figure 6b. In attempts to find a solution for this issue, we were able to turn this into an advantage by using them as cooling fins.

Another important aspect to consider was the position of the motor relative to the center of the aperture. To avoid chain release, position of the shaft of the driving chain wheel should be horizontal and up to $60^{\circ}$ as seen in Figure 6c. This approach reduces the necessity of chain tensioner or guide. However, the present design features a chain tensioner nonetheless to avoid problems in the long term. It should be noted that by manually releasing 8 nuts, the driving sprocket can be moved outwards and chain tension can be restored. Also, eight holes placed in the bottom plate of the iris and the reactor's flanges allow us to position the iris in eight different positions with a $45^{\circ}$ angle. 


\section{CLOSED LOOP CONTROL SYSTEM}

Development of a closed loop real time control system is challenged by the transient behavior of the incoming solar radiation. In present work, the control system is based on single input single output (SISO). The input is the difference (fault) between the set point value which is desired temperature inside the reactor and the feedback temperature which is process value. The output value is determined by a PID controller representing the aperture area. The output value and the necessary hardware drive the iris mechanism until the desired area is achieved. This loop is repeated to minimize the fault between the set point and feedback value.

\subsection{Hardware configuration}

The closed loop control system determines the variable aperture area depending on the input value and the set point. In physical terms, the control system is build of three main parts: the sensors, the controller and the drive train. The selection of each component has an influence on the accuracy, precision, reliability and speed. Figure 7 represents complete overview of the components used in present work.

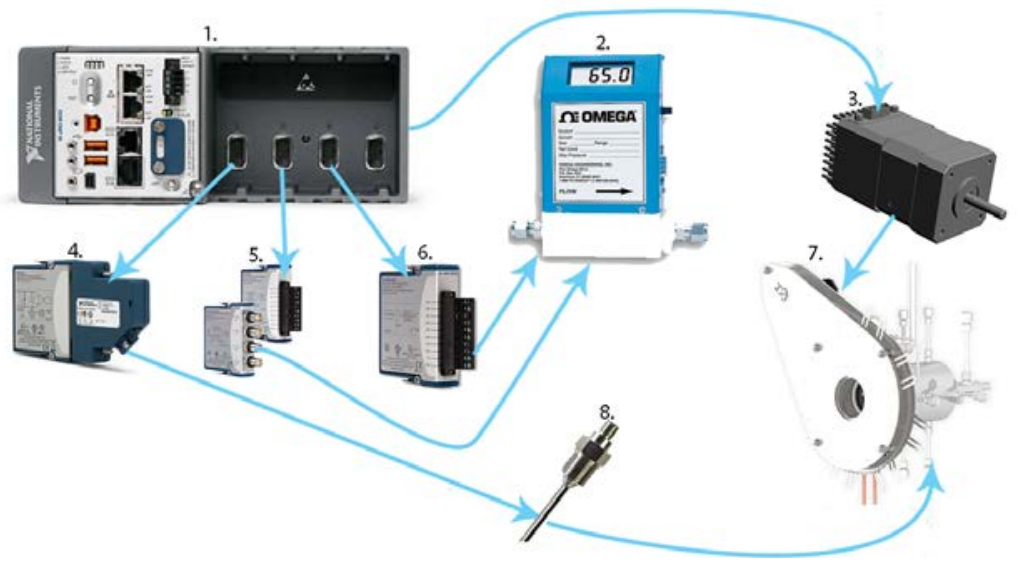

1 Compact RIO hardware platform

2 Mass flow controller

3 Stepper motor with integrated driver

and controller

4 16-channel thermocouple module

5 4-channel voltage output module

6 4-channel voltage input module

7 Iris mechanism

8 M12 K-type thermocouples

Fig. 7: Hardware setup and components of the control loop

The control design is based on temperature measurements inside the reactor measured by thermocouples. There are 9 K-type thermocouples embedded in reactor cavity to ensure reliable temperature readings and provide feedback on the cold and hot spots. 4 bolt-on thermocouples accompanied by 2 non-touchable thermocouples are used to provide feedback on the water-cooling and temperature of the iris mechanism. The last thermocouple is placed in the exhaust.

Feedstock control is done by the controller which sends steering signal to the mass flow controller. The mass flow controller opens and closes an electromagnetic valve depending on the input signal and then gives feedback to the main controller. Embedded systems from National Instruments provide real-time simulations, the motor control and data acquisition. Compact RIO hardware platforms are divided into three internal components called a hybrid system as follows: (1) the real-time controller, (2) the field programmable gate array (FPGA), and (3) the interchangeable c-modules. Communication between the real-time controller and the FPGA passes through PCI bus which provides reliable and fast data transport.

The c-modules are plugged in the chassis and may communicate with the real time controller and the FPGA. FPGA is a reconfigurable silicon chip made out of thousands of logical cells containing smaller hardware elements like flip-flops, lookup tables etc. Each of these logical cells includes several inputs and outputs. Developers are to configure the integrated circuit by use of hardware based software languages. The realtime controller and the implemented operating system are code/IP based to ensure easy data acquisition. Interchangeable c-modules offer great flexibility to the hardware platforms. The present test application is made of three c-modules: (1) Thermocouple module, (2) Analog voltage in module, and (3) Analog voltage out module. 
Translation of the control signal to real-time physical movements is accomplished by the stepper motor with integrated controller and driver. First, the relation between the aperture area and the angle is determined. Equation (10) will be implemented in the main controller to identify the amount of steps to have certain aperture area. The amount of steps will be sent and processed in the external controller. The driver will amplify the signal from the external controller and power the stepper motor, which physically moves the iris mechanism.

\subsection{Plant model}

LabVIEW was used to develop the model plant and control algorithms. The plant model and the control system were created and tested in the LabVIEW control and simulation add-on. The plant model and control algorithm will be programmed in the compact RIO for real-time simulations as soon as the hardware and iris mechanism are installed.

The temperature inside the reactors cavity is calculated by several thermodynamic factors. Energy balance for a solar reactor can be given as follows:

$$
\Delta \dot{\mathrm{E}}+\dot{\mathrm{E}}_{\text {reaction }}=\dot{\mathrm{E}}_{\text {absorbed }}-\dot{\mathrm{E}}_{\text {radiation }}-\dot{\mathrm{E}}_{\text {convection }}-\dot{\mathrm{E}}_{\text {conduction }}
$$

Convection and conduction losses throughout the solar reactor are assumed to be negligible to ease up the plant model. $\dot{E}_{\text {reaction }}$ denotes the energy usage for the chemical reaction, while $\Delta \dot{E}$ represents the energy disposable to achieve the set point temperature. $\dot{E}_{\text {absorbed }}$ is the absorbed energy and which can be expressed as follows:

$$
\dot{\mathrm{E}}_{\text {absorbed }}=P_{\text {aperture }} \cdot \alpha_{\text {eff }}
$$

where $P_{\text {aperture }}$ is the power intercepted by the variable aperture and $\alpha_{e f f}$ is absorption efficiency. $\dot{E}_{\text {radiation }}$ is

$$
\dot{\mathrm{E}}_{\text {radiation }}=\sigma \cdot \varepsilon_{\text {eff }} . \mathrm{A} \cdot T^{4}
$$

where $\varepsilon_{e f f}$ is the emissivity and $\sigma$ is the Stefan-Boltzmann constant given by $5.67 \times 10^{-8} \mathrm{~W} / \mathrm{m}^{2} \cdot \mathrm{K}^{4}$. While absorbed energy dependents on the power input and the surface area, the re-radiation losses vary with the surface area and temperature to the fourth power. Consequently, opening the iris mechanism does not always result in temperature rise as the re-radiation losses exceed absorbed energy. Starting from these equations and the conclusion of the experiments by the previous solar reactor done by our research group; the governing equations can be simplified into a first order system of the following form:

$$
\frac{d T_{r}}{d t}=\alpha \cdot\left(P_{i n} \cdot A-(\beta \cdot A+\gamma)\right) \cdot\left(T_{r}-T_{a m b}\right)
$$

where $\beta, \alpha$ and $\gamma$ are model specific constants and are obtained by experimental testing of the solar reactor. $A^{*} P_{\text {in }}$ represents the temperature increase caused by the flux generated from the solar simulator, and $\beta A\left(T_{r}-T_{a m b}\right)$ is the term associated with the energy loss whereas the term $\gamma\left(T_{r}-T_{a m b}\right)$ represents the reradiation losses. It is important to note that these constants are model specific and will be adjusted for the new solar reactor when testing is possible. For now, let us assume the following values: $\beta=0.3826, \alpha=$ 0.0002 and $\gamma=0.575$. 


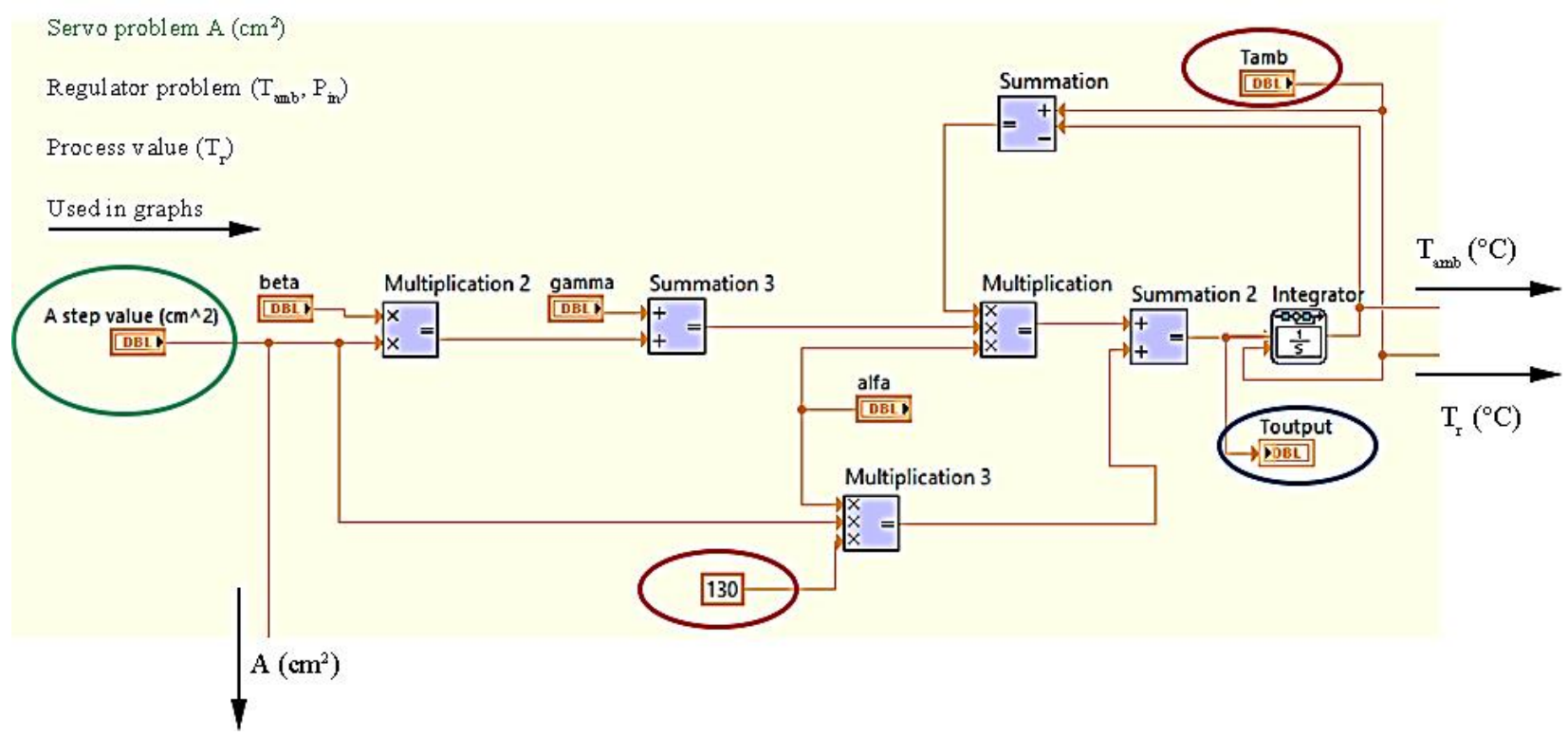

Fig. 8: Plant model

The following step is to implement this non-linear differential equation in LabVIEW. Figure 8 gives representation of Equation 4 in LabVIEW, which is used as a simulation plant for tests and tuning of the PI regulator. Figure 9 shows the front panel where the control values are assigned and date is exported.

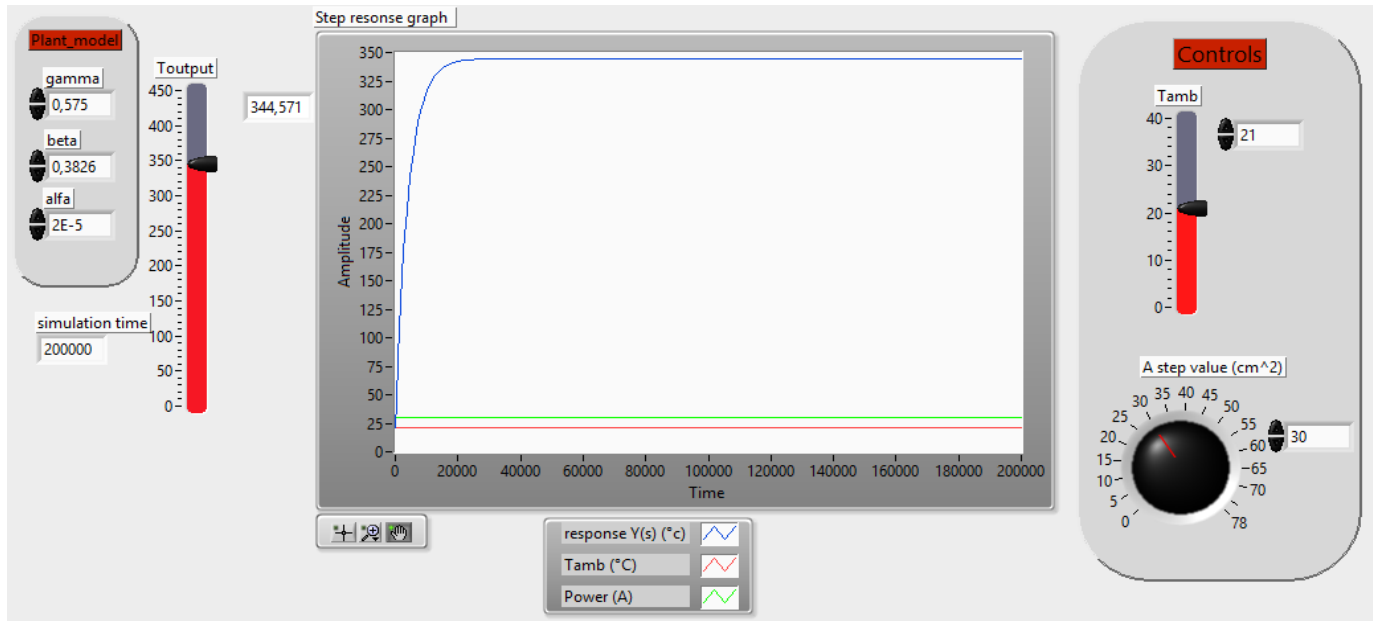

\subsection{PID controller}

Fig. 9: Model plant front panel

PID controller has three terms: a proportional, an integral and a derivative operation which are tuned according to the plant model. Figure 10 shows the control loop which is a PI regulator with an anti-windup for the integral term. Saturation block is implemented to limit the controller's output which in turn opens/closes the iris mechanism per limits. A rate limiter is used to limit the amount of change in opening or closing during certain timeframe. Because the thermocouples delay the system, implementation of transport delay is necessary. The power input is a sinusoidal function which represents the transient behavior of the solar radiation. The polynomial equation ensures the link between the stepper motor and aperture area. 


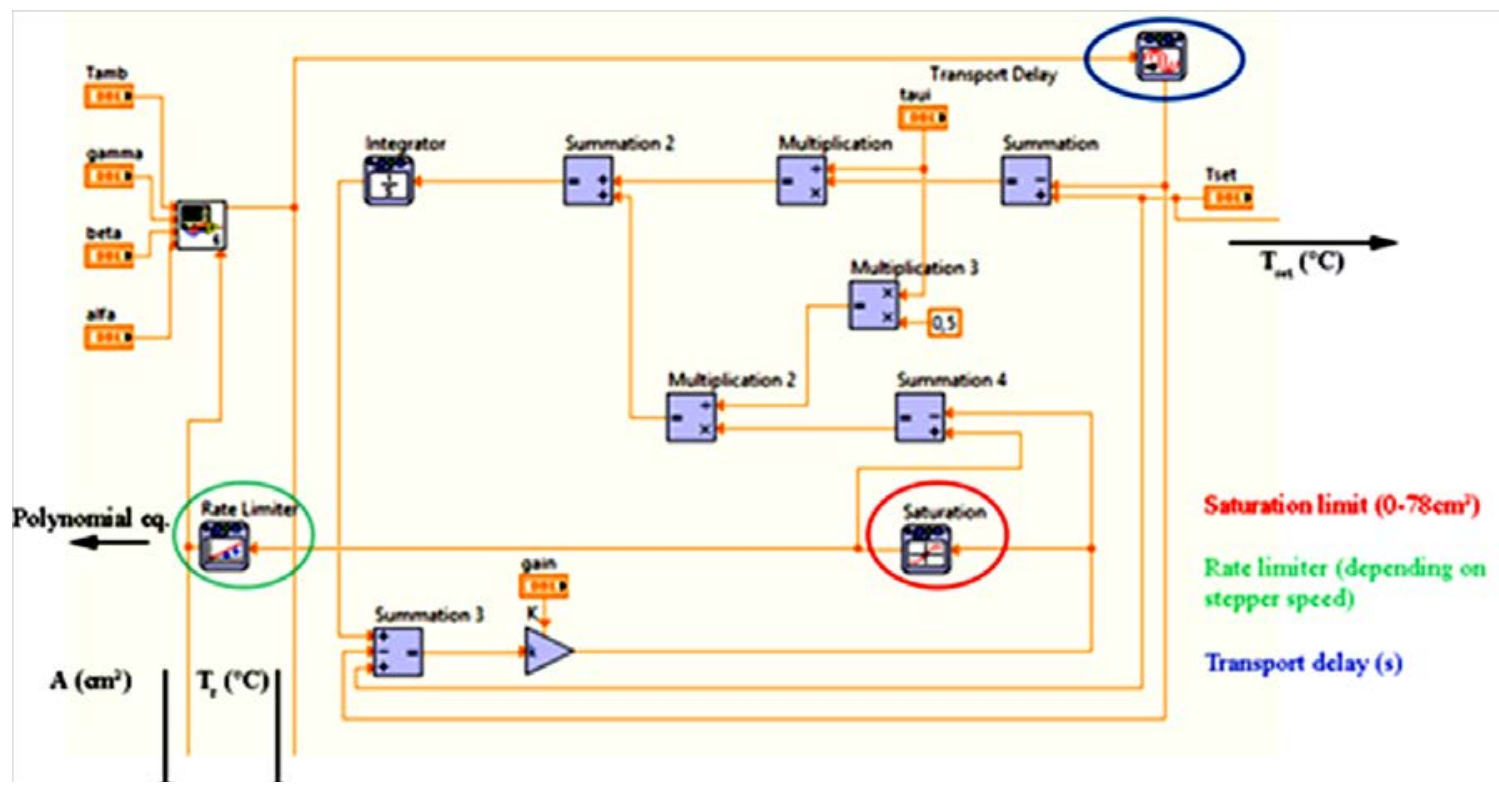

Fig. 10: Illustration of the PI control loop

A fine-tuned control system depends on several parameters such as overshoot, response time, stability etc. Tuning parameters for the simulation plant are derived from step response functions of the system. Because the differential equation to simulate the solar reactor's behavior is non-linear, the step needs to be settled nearby the operation point. By using the Cohen-coon control equation, following values are obtained: gain of 4588 and $\tau_{i}$ of 1317. Simulation of these control parameters is given in Figure 11. Control system successfully achieves the set point with negligible error and stability of the system is maintained until the end of simulation. The simulation is done with account of the transient nature of the sun. This is why the aperture area increases due to decrease of solar radiation at the end of the simulation.

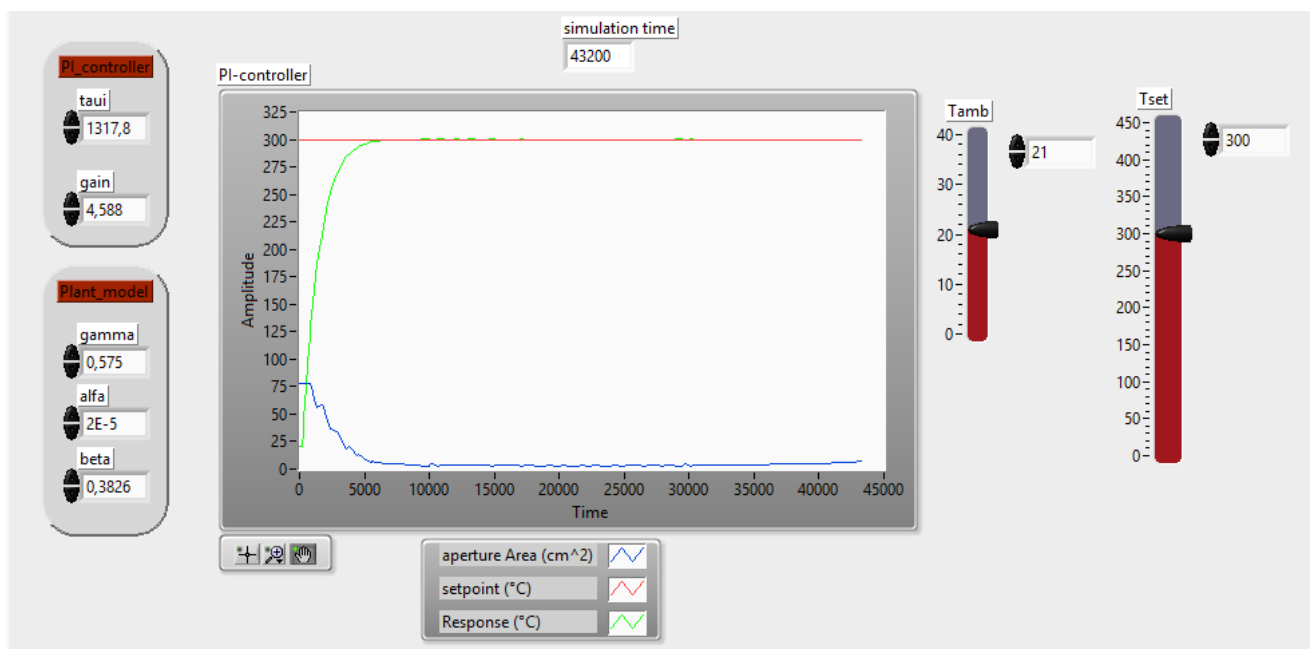

Fig. 11: PI controller front panel and simulation

Illustration of the planned experimental setup is shown in Figure 12a. Once the control system is implemented, first experiments will be done at room temperature in order to test and fine-tune the control system. Next step is to run the experiments using $7 \mathrm{~kW}$ adjustable power high flux solar simulator. 


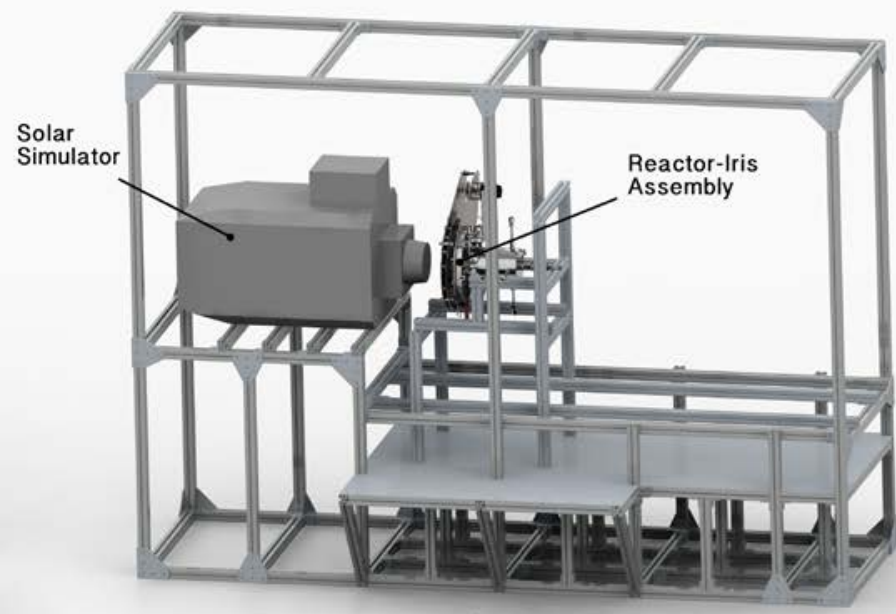

a

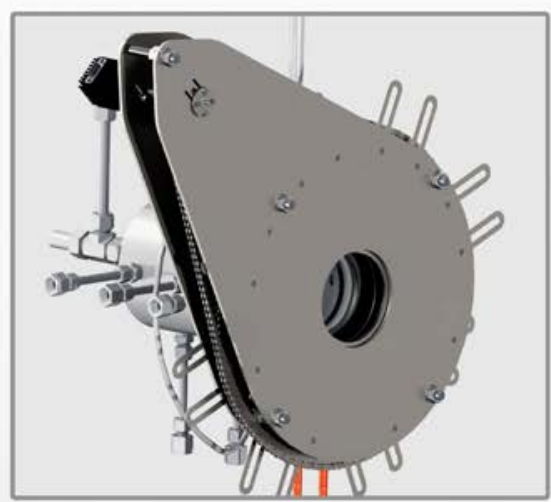

b

Fig. 12: (a) Experimental setup, (b) Reactor-Iris assembly

\section{ANALYTIC METHODOLOGY}

Before starting experiments, an analytic study of the iris design needs to be done. The key feature of the aperture mechanism is the opening area $A$, as this will regulate the incoming radiation flux $P_{\text {aperture }}$. The aperture surface area as a function of the motor's angular position must be calculated for accurate control of the stepper motor's rotation. Unlike other iris mechanisms where single-sided blades are being used, development of analytical relations for two-edge blades is very complex. For example, when single-sided blades are being used, the inner surface area of the iris can be approximated as an octagon throughout the whole operation cycle as illustrated in Figure 4e. However, two-blade iris mechanism has different geometrical shape as it opens and closes. As it is seen in Figure 13, the area is a circle in fully open position, then turns into an octagon, and then transforms into hexagon and then back to an octagon. This complicates the calculations to identify where the blades intersect and the development of analytical relations for the blades' axial displacement.
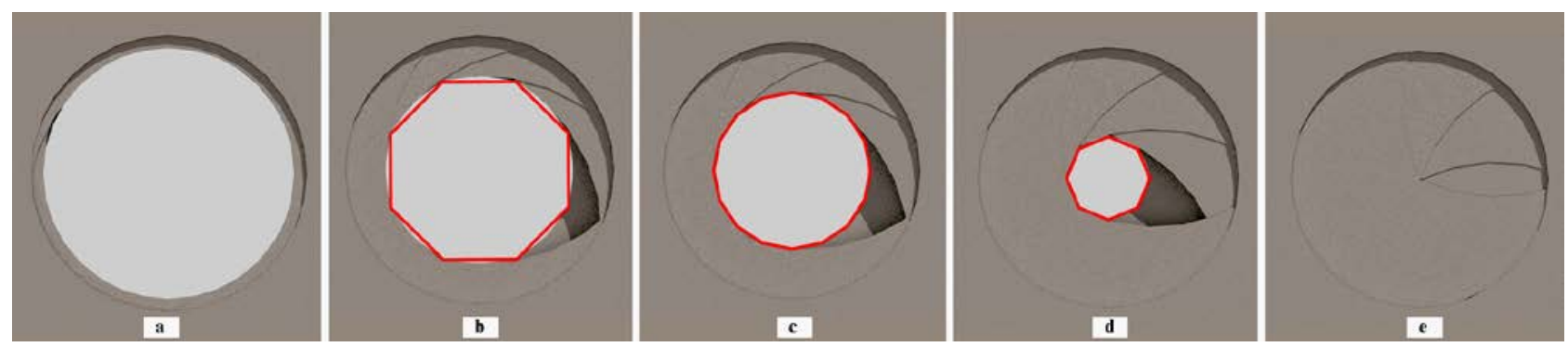

Fig. 13: Evolution of aperture area geometry as it closes

Because the aperture geometry changes, number of intersection points between two adjacent blades varies as well. In order to simplify this complexity, an assumption can be made approximating the geometry of the aperture. The simplest way of approximating the aperture's quasi-circular shape using analytical relations between the blades would be to measure the exact area using the CAD-software. This way, accuracy of the approximation depends on the number of measured points. In present work, measurements were done for several angles of the stepper motor $\theta$ with $5^{\circ}$ interval between each angular position. Then the points were plotted and fitted to a $6^{\text {th }}$ degree polynomial using Matlab, which gave the following equation:

$$
A=C_{1} \theta^{6}+C_{2} \theta^{5}+C_{3} \theta^{4}+C_{4} \theta^{3}+C_{5} \theta^{2}+C_{6} \theta+C_{7}
$$

where $C_{1}=1.064 \mathrm{e}^{-10}, C_{2}=-1.098 \mathrm{e}^{-8}, C_{3}=2.632 \mathrm{e}^{-7}, C_{4}=-8.499 \mathrm{e}^{-4}, C_{5}=-0.128, C_{6}=2.166$ and $C_{7}=7874.978$. 
An alternative approach would be through analytic calculations. The motion simulations have indicated that the intersection point between the two blades shifted relatively over $90^{\circ}$ angle is the best option to approximate the area. Figure 14 illustrates the reference for analytical relation developed in present work.

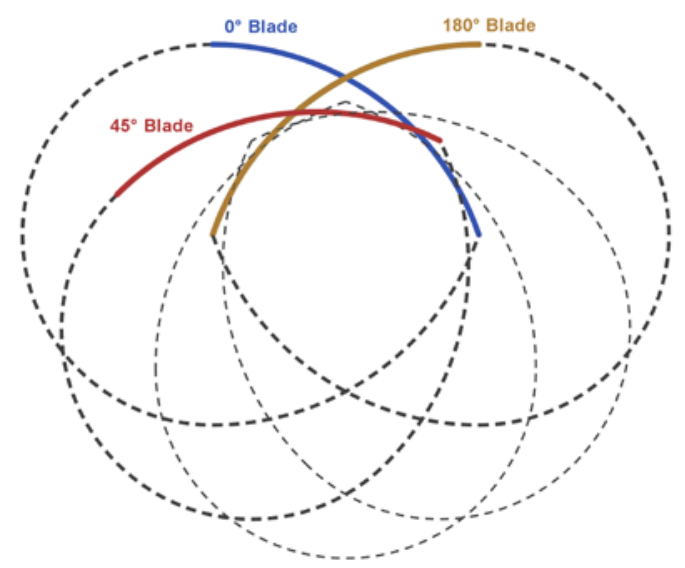

$\mathbf{a}$

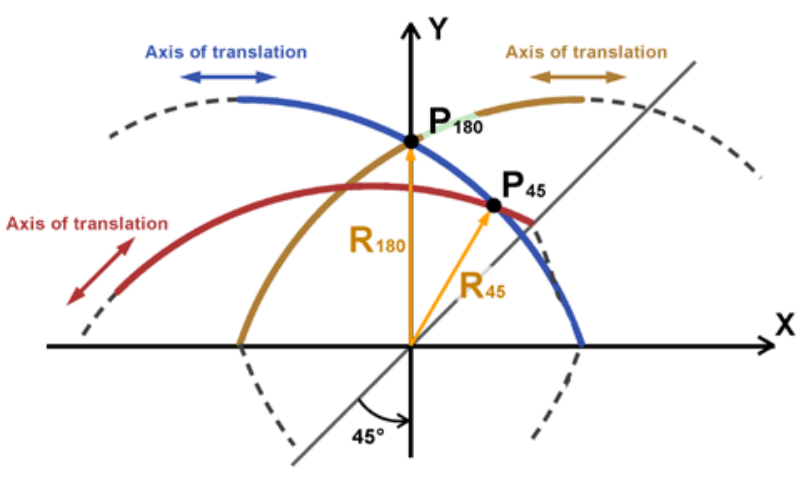

b

Fig. 14: (a) Intersection pattern of the blades, (b) Intersection points of $45^{\circ}$ and $180^{\circ}$ shifted blades

The set of parametric equations, derived from the equation of a circle, defining the intersection point $\mathrm{P}_{180}$ of the first pair is as follows:

$$
P_{180}(X, Y)\left\{\begin{array}{c}
x=0 \\
y=\sqrt{74^{2}-d^{2}}-24
\end{array}\right.
$$

where " $d$ " is the displacement of the blade and $\mathrm{P}_{180}$ is the intersection point of two blades with $180^{\circ}$ angular shift.

Analytical relations for the blades' axial displacement can be developed by studying actuator's pin-slot mechanism. The translation " $d$ " could then be written as a function of the angular position of the stepper motor $\theta$ as follows:

$$
d=\frac{154 \sin (0.085 \theta)}{\cos (0.085 \theta)-0.97 \sin (0.085 \theta)}
$$

As the coordinates of the intersection point $\mathrm{P}_{180}$ (Figure 14b) are defined by Equations (6), the area $\mathrm{A}_{180}$ can be written as:

$$
A_{180}=\pi\left(\sqrt{74^{2}-(111 \sin (\alpha) \csc (\alpha+2.33854))^{2}}-24\right)^{2}
$$

where $\alpha=0.085(\pi / 180) \theta$.

Intersection point $\mathrm{P}_{45}$ and surface area $\mathrm{A}_{45}$ can be determined analogous to point $\mathrm{P}_{180}$ in Equation (6) and surface area $\mathrm{A}_{180}$ in equation (8). Figure 15 gives these results showing the surface area $A_{i}$ as a function of stepper motor angle $\theta$. 


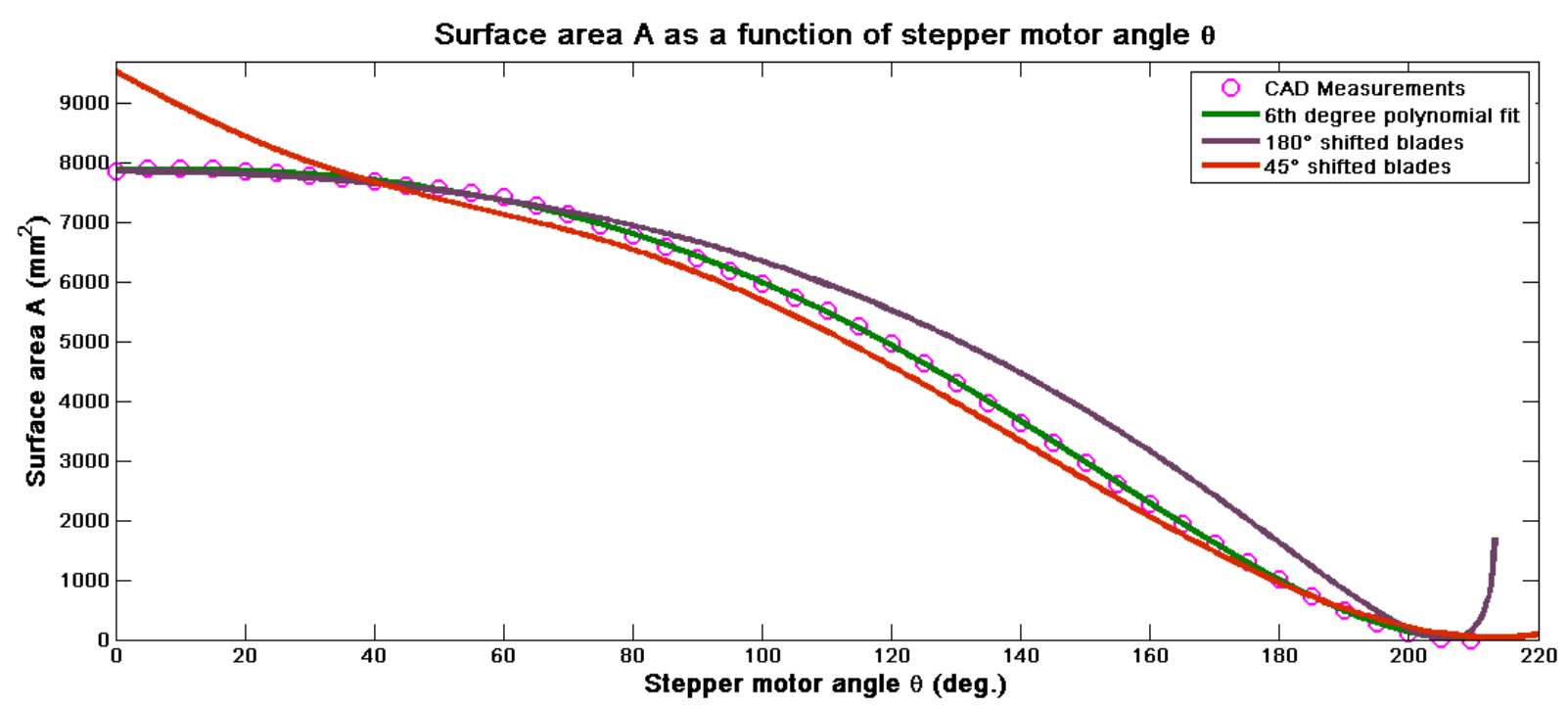

Fig. 15: Plot of the surface area $A$ as a function of the angular position of the stepper motor

From this plot, it may be concluded that for the best mathematical approximation of the surface area, the approximated surface area should be split in two domains of definition. The $180^{\circ}$-curve comes very near to the polynomial initially. At around 40 degrees of stepper motor angle, the $45^{\circ}$-curve intersects both of the other curves and then approximates the polynomial relatively better than the $180^{\circ}$-curve. The final curve describing the surface area $A$ mathematically with accompanying domains of definition can be expressed as:

$$
A(\theta)=\left\{\begin{array}{c}
A_{180}(\theta), 0<\theta \leq 40.7 \\
A_{45}(\theta), 40.7<\theta
\end{array}\right.
$$

Starting from the best approximation of the inner surface area, being the polynomial Equation (5), the relation can then be integrated in LabVIEW. During the experiments the aperture will be measured gradually to quantify the actual surface area. This then provides the ability to compare the empirical with both of the theoretical relationships and evaluate these. Possible errors can be quantified and applied in correctional relationships.

The surface area as a function of the stepper motor angle being one part, another aspect of the theoretical methodology includes the force and torque analysis of the mechanism. The rated torque delivered by the stepper motor can be associated with the forces applied to the mechanism. This initial torque is transferred to the actuator which then converts torque in forces that make the blades slide in- and outwards. Figure 16 demonstrates the resulting forces and torque for the closing motion of the aperture. It should be noted that for the following elaboration, some assumptions were made. First, the chain was supposed to be tensioned. This means there is no sagging and the chain can be seen as a line tangent to both pitch circles of the gears. Secondly, because of the metal-to-metal contact and minor thickness of the blades and actuator, there are no frictional forces. In addition, any form of clearance was neglected which implies that there is no backlash in the chain. 


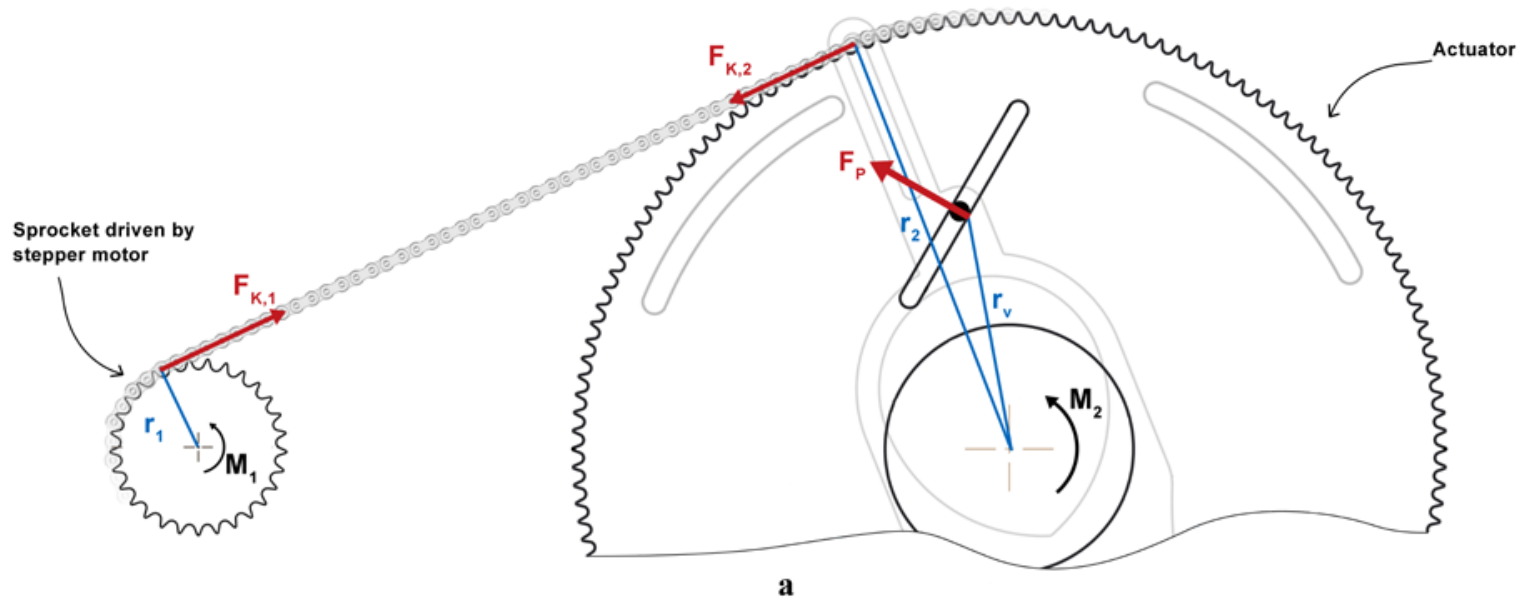

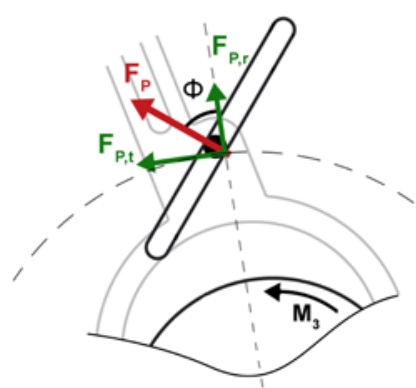

b

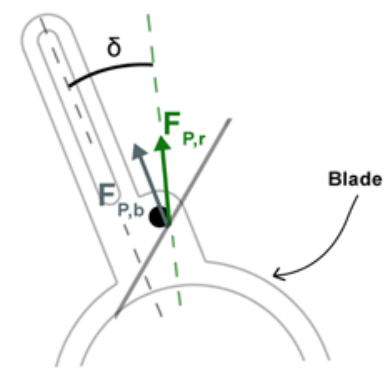

c

Fig. 16 (a) Chain drive force and torque transfer, (b) Pin-slot forces, (c) Resulting movement force

Figure 16a illustrates the tension forces on the chain; $F_{K, 1}$ and $F_{K, 2}$. The counteracting chain force $F_{K, 1}$ is generated by the stepper motor's torque $M_{1}$ and is equal to $F_{K, 2}$. The driving torque $M_{1}$ is transmitted to the actuator plate in proportion to the gear ratio:

where $\frac{R_{1}}{R_{2}}=\frac{1}{i}=0.193$.

$$
M_{1}=\frac{R_{1}}{R_{2}} * M_{2}
$$

When the actuator rotates, the pins of each blade experience a reacting force $F_{P}$. This force can be split in a radial and a tangential components; $F_{P, r}$ and $F_{P, t}$. The moment of force $M_{3}$ can then be formulated as a function of $F_{P, t}$ :

$$
M_{3}=F_{P, t} * r_{v}
$$

where $r_{v}$ is the variable distance to the pin-slot contact point. With $F_{P, t}=F_{P, r} * \tan (\Phi)$, Equation (11) can be written as:

$$
M_{3}=\mathrm{F}_{P, r} * \tan (\Phi) \cdot r_{v}
$$

$F_{P, r}$ multiplied with the cosine of angle $\delta$ gives the resulting force $F_{P, B}$ for each blade. The final relation of the mechanism, represented in Figure 17 gives the motor's torque as a function of the resulting force $F_{P, B}$ that leads to the translational movement of each blade. For symmetry reasons the overall torque of the actuator $M_{2}$ is equal to the sum of the moments for each one of the blades $M_{3}$. Since there are eight blades this gives $M_{2}=8 * M_{3}$. Substituting this into Equation (12), results in:

$$
M_{1}\left(F_{P, B}\right)=1.55 *\left(r_{v} \tan \phi\right) *\left(\frac{F_{P, B}}{\cos (\delta)}\right)
$$

with $\delta=14^{\circ} ; r_{v}$ and $\Phi$ are variable parameters depending on the blade pin's position in the slot. 


\section{CONCLUSIONS}

A new iris design for a solar reactor was presented along with analytical relations correlating the aperture area as a function of stepper motor's angular rotation. For each insolation level, aperture area of different size is required to accommodate the fluctuation in solar radiation. As the aperture diameter is increased, the average gas temperature is expected to increase until it reaches a maximum for an optimum diameter. Beyond that optimum value, re-radiation losses become higher although more power is intercepted by the aperture. Because for a particular time of the day, depending on the magnitude of direct normal insolation, there exists an optimum aperture size that can maintain the desired level of temperature inside the reactor; selecting an optimum aperture size for a particular time of the day is a compromise between maximizing radiation capture and minimizing re-radiation losses. Proposed iris mechanism may address this by offering variable area opening. One main feature of the iris mechanism presented in this work is the utilization of two-sided blades. This feature results in an improved permanent circularity of the aperture area.

A closed loop control system was designed to control the reactor temperature as the iris opens/closes based on temperature readings inside the reactor. The motor control and data acquisition was designed by using embedded systems of National Instruments providing real-time simulations. Compact RIO was implemented in the system. Communication between the real-time controller and the FPGA was made through PCI bus. Translation of the control signal to real-time physical movements was planned to be done by the stepper motor with integrated controller and driver. The relation between the aperture area and the angle was implemented in the main controller to identify the amount of steps to have certain aperture area. The driver was implemented to amplify the signal from the external controller and power the stepper motor to moves the iris mechanism. A PID controller was designed and a model plant was built. It was observed by simulations that the control system successfully achieved the set point with negligible error. The simulation was done by accounting the transient nature of the sun.

As future work, the aforementioned aperture design will be manufactured and experimentally tested. Data acquisition will enable us to correlate theoretical relationships with these measured values. Model plant modifications will be carried out as to accommodate the behaviour of the new plant. In sync with the steadystate research, the PID controller will be further tuned and the plant model will be compared with actual behaviour of the system.

\section{ACKNOWLEDGMENT}

This research has been funded by the KU Leuven internal Impulse Fund. We would like to express our appreciation to Dr. Gorik de Samblanx, Dr. Patrick van Raymenant, and Dr. Filip Logist for their assistance in several aspects of the ongoing work.

\section{NOMENCLATURE}

\begin{tabular}{|c|c|c|c|c|c|}
\hline$P_{\text {aperture }}$ & $\begin{array}{l}\text { Incoming power caught by } \\
\text { the aperture }\end{array}$ & $(W)$ & $R$ & Radius of the aperture & $(\mathrm{mm})$ \\
\hline$\alpha_{\text {eff }}$ & Effective absorptance & $(-)$ & $\dot{\mathrm{E}}_{\text {reaction }}$ & $\begin{array}{l}\text { Energy dissipation by the } \\
\text { chemical reaction }\end{array}$ & (J) \\
\hline$\sigma$ & Stefan-Boltzman constant & $(-)$ & $\Delta \dot{\mathrm{E}}$ & $\begin{array}{l}\text { Thermal energy left for } \\
\text { temperature increase }\end{array}$ & (J) \\
\hline$A$ & Aperture surface area & $\left(m^{2}\right)$ & $\dot{\mathrm{E}}_{\text {absorbed }}$ & $\begin{array}{l}\text { Thermal energy absorbed by } \\
\text { the reactor }\end{array}$ & (J) \\
\hline$T_{r}$ & $\begin{array}{l}\text { Temperature inside the } \\
\text { reactor cavity }\end{array}$ & $(\mathrm{K})$ & $\dot{\mathrm{E}}_{\text {radiation }}$ & Re-radiation losses & (J) \\
\hline$P_{\text {in }}$ & Incoming solar power & (A) & $\dot{\mathrm{E}}_{\text {convection }}$ & Convection losses & (J) \\
\hline$T_{a m b}$ & Room temperature & (K) & $\dot{\mathrm{E}}_{\text {conduction }}$ & Conduction losses & (J) \\
\hline$\theta$ & $\begin{array}{l}\text { angular position of stepper } \\
\text { motor }\end{array}$ & $\left({ }^{\circ}\right)$ & $\gamma, \alpha, \beta$ & Plant specific parameters & $(-)$ \\
\hline$d$ & Blade’s displacement & $(\mathrm{mm})$ & $\tau_{\mathrm{i}, \text { gain }}$ & $\begin{array}{l}\text { Integration and proportional } \\
\text { constant (PI) }\end{array}$ & $(-)$ \\
\hline$F_{K, i}$ & Tension force on the chain & $(\mathrm{N})$ & $M_{i}$ & Torque or moment of Force & $(\mathrm{Nm})$ \\
\hline
\end{tabular}




\begin{tabular}{|c|c|c|c|c|}
\hline$r_{1,2}$ & $\begin{array}{l}\text { Radius of (1) sprocket and } \\
\text { (2) actuator }\end{array}$ & $(\mathrm{mm})$ & $i$ & gear ratio \\
\hline$F_{P}$ & Pin force & $(\mathrm{N})$ & $F_{P, t}, F_{P, r}$ & $\begin{array}{l}\text { Tangential and radial } \\
\text { components of } F_{p}\end{array}$ \\
\hline & Variable distance to pin & $(\mathrm{mm})$ & $\Phi$ & Angle between $F_{P}$ and $F_{P, r}$ \\
\hline$F_{P, B}$ & $\begin{array}{l}\text { Resulting translational } \\
\text { force }\end{array}$ & $(\mathrm{N})$ & $\delta$ & Angle between $F_{P, r}$ and $F_{P, B}$ \\
\hline
\end{tabular}

\section{REFERENCES}

[1] Bernecker, D., Reiss, C., Angelopoulou, E., Hornegger, J., “Continuous short-term irradiance forecasts using sky images,” Solar Energy., 110, pp. 303-315, (2014).

[2] Charvin, P., Abanades, S., Neveu, P., Lemont, F., Flamant, G., "Dynamic modeling of a volumetric solar reactor for volatile metal oxide reduction,” Chemical Engineering Research And Design., 86, pp. 1216-1222, (2008).

[3] Petrasch, J., Osch, P., Steinfeld, A., “Dynamics and control of solar thermochemical reactors,” Chemical Engineering Journal., 145, pp. 362-370, (2009).

[4] Saade, E., Bingham, C., E. Clough, D., W. Weimer, A., "Dynamics of a solar-thermal transport-tube reactor," Chemical Engineering Journal., 213, pp. 272-285, (2012).

[5] Saade, E., Bingham, C., E. Clough, D., W. Weimer, A., “Model predictive control of a solar-thermal reactor,” Solar Energy., 102, pp. 31-44, (2014).

[6] Ozalp, N., Jayakrishna, D., "Numerical study on the thermal interaction of gas-particle transport for a vortex flow solar reactor.” ASME 4th International Conference on Energy Sustainability, vol. 2, ES2010-90325, pp. 133-141 (2010).

[7] M. Besarati, S., Goswami, D.Y., K. Stefanakos, E., "Optimal heliostat aiming strategy for uniform distribution of heat flux on the receiver of a solar power tower plant,” Energy Conversion and Management., 84, pp. 234-243, (2014).

[8] J.P. Sack, M. Roeb, C. Sattler, R. Pitz-Paal, A. Heinzel., "Development of a system model for a hydrogen production process on a solar tower," Solar Energy., 86, pp. 99-111, (2012).

[9] Abdulla, S., Zahreddine, H., El Zamli, M., Majid, F.A., Rizk, I., Al Hamidi, Y., Ozalp, N., “Design, manufacturing and testing of a camera-like aperture mechanism for a solar reactor," Proc. of the ASME 2011 Int. Mechanical Engineering Congress \& Exposition., vol. 3, IMECE2011-65814, pp. 305-319, (2011)

[10] Al Hamidi, Y., Abdulla, S., El Zamli, M., Rizk, I., Ozalp, N., "Design, manufacturing and testing of an aperture mechanism for a solar reactor,” Proc. of the ASME $5^{\text {th }}$ Int. Conf. on Energy Sustainability., parts A,B \& C, ES2011-54567, pp. 1661-1672, (2011).

[11] Yamada, T., Kosako, K., Ohsawa, Y., “Diaphragm control apparatus of interchangeable lens camera,” U.S. Patent Application No. 13/737,202, (2013).

[12] Cheung, N., "Virtual variable valve intake and exhaust for the internal combustion engine," U.S. Patent Application No. 12/156,928, (2012).

[13] Gat, N., Zhang, J., “Continuous variable aperture for forward looking infrared cameras based on adjustable blades,” U.S. Patent Application NO. 13/454,006, (2013).

[14] Jong-hyeon, C., Kyu-Dong, J., Eunsung, L., Minseog, C., Seungwang, L., Woonbae, K., "Variable aperture controlled by microelectrofluidic iris,” Optic Letters, Vol. 39 (15), pp. 2919-2922, (2013).

[15] Draheim, J., Burger, T., Korvinck, J.G., Wallrabe, U., "Variable aperture stop based on the design of a single chamber silicone membrane lens with integrated actuation,” Optic Letters. Vol. 36 (11), pp. 2032-2034, (2011).

[16] Syms, R., Zou, H., Stagg, J., Veladi, H., “Sliding-blade MEMS iris and variable optical attenuator.” Journal of Micromechanics and Microengineering, 14, pp. 1700-1710, (2004). 